\title{
Edible Fruits: Beyond Nutritional Value
}

\author{
Lalith Jayasinghe*
}

Institute of Fundamental Studies, Hantana Road, Kandy, Sri Lanka; E-mail: ulbj2003@yahoo.com

Fruits play a prominent role in the human diet and are known to be rich in essential vitamins, simple sugars, fiber, minerals, micronutrients which are essential factors to maintain good health. Apart from their traditional uses many fruits have useful medicinal properties for curing diseases and disorders including cancer, diabetes, coronary heart diseases etc. Most studies on edible fruits are limited to their nutritional properties. Edible fruits have been consumed for thousands years and should have low toxicological issues associated with their use. Therefore fruits are a promising source for the identification of environmentally friendly bioactive compounds. During the last few years we have carried out extensive studies to explore the possibilities of utilizing edible fruits as a source for bioactive compounds with special reference to their antioxidant, antifungal, brine shrimp lethality, phytotoxic and enzyme inhibitory activities, which are important in food supplements, pharmaceutical preparations and in agricultural crop protection. Enzyme inhibition bioassays and the discovery of new naturally occurring enzyme inhibitors are important in the field of drug research. Studies on the popular edible fruits of Sri Lanka, Aegle marmelos, Artocarpus altilis, Averrhoa carambola, Flacourtia indica, Flacourtia inermis, Garcinia cambogia led to the isolation and structure elucidation of various classes of compounds including some novel natural products with some interesting bioactivities.

Keywords: Edible fruits, Bioactive natural products. 